\title{
Conservação do sêmen e liquefação do coágulo seminal de macaco-prego (Cebus apella) em água de coco em pó (ACP-118 ${ }^{\circledR}$ ), em diferentes temperaturas
}

\author{
Semen conservation and seminal clot liquefaction of capuchin monkey (Cebus apella) in powdered \\ coconut water extender (PCW) at different temperatures
}

\author{
Karol Guimarães Oliveira' ${ }^{\mathrm{I}, \mathrm{II}, \mathrm{III}{ }^{*}}$ Paulo Henrique Gomes de Castro ${ }^{\mathrm{III}}$ \\ José Augusto Pereira Carneiro Muniz ${ }^{\mathrm{III}}$ Sheyla Farhayldes Souza Domingues ${ }^{\mathrm{I}, \mathrm{II}}$
}

RESUMO

O objetivo do estudo foi avaliar a água de coco em pó (ACP) na conservação do sêmen e liquefação do coágulo seminal de Cebus apella. O sêmen de seis machos adultos foi coletado por eletroejaculação (EEJ), diluído em solução à base de ACP-118 ${ }^{\circledR}$ e submetido à incubação em banho-maria a 33, 35 e $37^{\circ} \mathrm{C}$, por 24 horas. Avaliou-se a integridade espermática por meio da coloração eosina-nigrosina a cada uma hora durante as seis horas iniciais e após 24 horas de incubação. Os volumes médios e as concentrações espermáticas

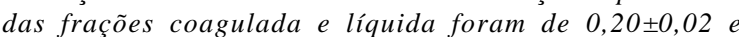
$0,20 \pm 0,10 \mathrm{~mL} ; 1,1 \pm 0,3 \times 10^{8}$ e $1,3 \pm 0,9 \times 10^{7}$ espermatozoides $\mathrm{mL}^{-1}$, respectivamente. Somente em uma amostra da fração líquida foram verificados espermatozoides com motilidade (20\%) e vigor (4), perdurando por 40 minutos. A maior parte do coágulo liquefez em ACP-118 ${ }^{\circledR}$ após 12 horas de incubação. $O$ melhor tratamento observado foi sob $33^{\circ} \mathrm{C}$, por manter até $47 \pm 12,8 \%$ de espermatozoides vivos após 24 horas. Concluise que o diluente à base de ACP é eficiente na liquefação do coágulo seminal e na manutenção da integridade espermática até 24 horas após a EEJ, nas temperaturas de 33, 35 e $37^{\circ} \mathrm{C}$.

Palavras-chave: Cebus apella, eletroejaculação, água de coco em pó, coágulo seminal.

\section{ABSTRACT}

The aim of this study was to evaluate the powdered coconut water $(P C W)$ in the semen conservation and seminal clot liquefaction. The semen of six adult male Cebus apella was collected by electroejaculation (EEJ), diluted in ACP-118 ${ }^{\circledR}$ extender and stayed in water bath at 33, 35 and $37^{\circ} \mathrm{C}$ for 24 hours. The sperm integrity was evaluated by eosin-nigrosine staining every one hour during the six initial hours and after 24 hours of incubation. The average volumes and sperm concentrations of clotted and liquid fractions were $0.20 \pm 0.02$ and $0.20 \pm 0.10 \mathrm{~mL}, 1.1 \pm 0.3 \times 10^{8}$ and $1.3 \pm 0.9 \times 10^{7}$ sperm $\mathrm{mL}^{-1}$, respectively. Immediately after collection, only in a sample of liquid fraction was observed $20 \%$ motility and vigor 4 , which stopped after 40 minutes. Most of the clot was liquefied in ACP$\mathbf{1 1 8}^{\circledR}$ after 12 hours of incubation. The best observed treatment was $33^{\circ} \mathrm{C}$, because it kept $47 \pm 12.8 \%$ of sperm integrity after 24 hours. It was concluded that the PCW extender is effective in the liquefaction of seminal clot and maintenance of sperm viability 24 hours after the EEJ at 33, 35 and $37^{\circ} \mathrm{C}$.

Key words: Cebus apella, electroejaculation, powdered coconut water, seminal clot.

\section{INTRODUÇÃO}

A biodiversidade mundial está mais ameaçada do que em qualquer outro período histórico (IUCN, 2008) e sua manutenção precisa ser assegurada para preservar a vida de espécies em risco de extinção, seja por meio de sua proteção nos habitats naturais (in situ), seja pela formação de bancos de germoplasma (exsitu) (DOMINGUES \& CALDAS-BUSSIERE, 2006).

O primata Cebus apella (macaco-prego) não figura entre essas espécies, sendo um excelente modelo experimental para o estudo da fisiologia reprodutiva (DOMINGUES et al., 2007; ARAÚJO et al., 2009) e da espermatogênese (LEÃO et al., 2008) e para o desenvolvimento de biotecnologias da reprodução a serviço de primatas neotropicais ameaçados de extinção (DOMINGUES \& CALDAS-BUSSIERE, 2006). Entretanto, técnicas como criopreservação de gametas,

\footnotetext{
IPrograma de Pós-graduação em Ciência Animal, Núcleo de Ciências Agrárias e Desenvolvimento Rural, Universidade Federal do Pará (UFPA), 66075-110, Belém, PA, Brasil. E-mail: karol bio@yahoo.com.br. *Autor para correspondência.

ILaboratório de Biologia e Medicina de Animais Silvestres da Amazônia, UFPA, Castanhal, PA, Brasil.

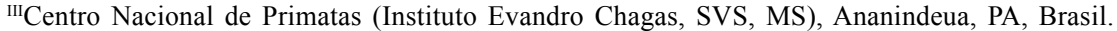


inseminação artificial, produção e transferência de embriões ainda não são aplicadas em $\boldsymbol{C}$. apella. Elas possuem o seu impacto limitado em razão da dificuldade de obtenção de espermatozoides viáveis (DOMINGUES \& CALDAS-BUSSIERE, 2006), pois o sêmen dessa espécie é formado por uma fração líquida e uma coagulada de difícil manipulação (NAGLE \& DENARI, 1983).

Trabalhos anteriores realizados por este grupo de pesquisa demonstraram a eficiência da água de coco in natura $(\mathrm{ACIN})$ a $37^{\circ} \mathrm{C}$ para a liquefação completa do coágulo seminal e manutenção de espermatozoides vivos até sete horas após a coleta do sêmen (ARAÚJO et al., 2009). Entretanto, o diluente à base de ACIN apresenta algumas desvantagens, como a dificuldade de manutenção da osmolaridade e do $\mathrm{pH}$, a disponibilidade limitada dos frutos em determinadas áreas e as diferenças físico-químicas entre frutos de regiões ou épocas distintas. Dessa forma, desenvolveuse um meio diluente à base de água de coco em pó $\left(\mathrm{ACP}^{\circledR}\right)$ que foi testado com sucesso em cães domésticos (CARDOSO, 2005). Portanto, os objetivos deste estudo foram a) avaliar a eficiência da ACP-118 ${ }^{\circledR}$, específica para o sêmen de C. apella, na manutenção da integridade espermática durante 24 horas de incubação realizada em banho-maria, nas temperaturas de 33,35 e $37^{\circ} \mathrm{C} \mathrm{e} \mathrm{b)} \mathrm{verificar} \mathrm{sua} \mathrm{eficácia} \mathrm{na} \mathrm{liquefação}$ do coágulo seminal.

\section{MATERIAL E MÉTODOS}

Local de execução

O estudo foi realizado no Centro Nacional de Primatas (CENP), órgão da Secretaria de Vigilância em Saúde, pertencente ao Ministério da Saúde, localizado na cidade de Ananindeua, Pará, Brasil.

\section{Animais}

O grupo experimental foi composto por seis machos adultos de Cebus apella, com idades estimadas entre 15 e 20 anos, selecionados pelo seu bom estado de saúde, confirmado por análises clínicas de rotina e características fenotípicas normais para a espécie, e cada animal foi submetido a uma avaliação prévia da consistência, forma, temperatura, simetria e mobilidade dos testículos e epidídimos, bem como da ausência de defeitos no escroto, conforme descrito por ARAÚJO et al. (2009). Os animais foram mantidos em gaiolas individuais de alumínio com dimensões de 80x90x80cm, dentro de galpões telados, sujeitos ao fotoperíodo natural, com alimentação diária à base de frutas, verduras, legumes, tenébrios (Zophobas morio), ração peletizada (FOXY Junior Supreme, 28\% proteína bruta;
PROVIMI, São José dos Pinhais, PR, Brasil) e água ad libitum.

\section{Procedimentos com os animais}

Os animais foram submetidos à contenção química, com a administração intramuscular da associação de Cloridrato de Ketamina (10 $\left.\mathrm{mg} \mathrm{kg}^{-1}\right)$ e Cloridrato de Xilazina ( $1 \mathrm{mg} \mathrm{kg}^{-1}$ ) (Laboratórios Köning S.A., Avellaneda, Argentina). Posteriormente, realizouse a higienização da região pubiana e do saco escrotal, com água destilada, sabão neutro líquido (Samira Indústria e Comércio de Produtos de Limpeza Ltda., Rio de Janeiro, RJ, Brasil) e solução fisiológica, seguida pela tricotomia, feita com lâmina estéril descartável. Essa etapa objetivou reduzir a quantidade de impurezas no sêmen após a coleta.

Foram realizadas oito coletas de sêmen por eletroejaculação (EEJ), com eletroejaculador à bateria (DUBOI, Campo Grande, MS, Brasil), equipado com uma sonda retal bipolar com $9 \mathrm{~mm}$ de diâmetro e $15 \mathrm{~cm}$ de comprimento, composta por dois eletrodos longitudinais, adaptado com um dispositivo de acionamento manual dos estímulos elétricos, garantindo maior controle e precisão. A sonda era previamente lubrificada com pomada de penicilina e dihidroestreptomicina (Ganadol ${ }^{\circledR}$ - Laboratório Fort Dodge Saúde Animal Ltda, Campinas, SP, Brasil) e posicionada ventralmente no reto, com introdução de $5 \mathrm{~cm}$ a partir do esfíncter anal. A estimulação consistia em quatro sessões com cinco séries de 20 estímulos elétricos crescentes de 25; 50; 75 e 100mA, e intervalo de 30 segundos entre cada série, conforme descrito por ARAÚJO et al. (2009). O pênis foi posicionado dentro do microtubo contendo $0,5 \mathrm{~mL}$ da solução diluente. Cada animal foi manipulado em intervalos mínimos de 30 dias entre os procedimentos de EEJ.

\section{Preparo do diluente}

O diluente, ACP- $118^{\circledR}$ (ACP Biotecnologia ${ }^{\circledR}$, Fortaleza - Ceará, Brasil), foi fornecido em envelopes plásticos individuais com $1,37 \mathrm{~g}, 300 \mathrm{mOsm} \mathrm{kg}^{-1} \mathrm{e} \mathrm{pH}$ 7,8 . Após o preparo, a solução $(1,37 \mathrm{~g}$ de ACP$118^{\circledR}+25 \mathrm{~mL}$ de água ultra pura) foi fracionada em alíquotas de $0,5 \mathrm{~mL}$ e mantidas em banho-maria até a estabilização da temperatura a 33,35 ou $37^{\circ} \mathrm{C}$, até o momento da coleta.

\section{Processamento do sêmen}

As frações líquida e coagulada foram depositadas separadamente nos microtubos contendo o diluente $(0,5 \mathrm{~mL})$ pré-aquecido de acordo com a temperatura analisada. Imediatamente após a coleta, as amostras eram acondicionadas em banho-maria 
(Modelo Digital BM-02, Marca Kacil, Brasil), perfazendo um total de 24 horas. As características macroscópicas como aspecto, cor e volume das frações líquida e coagulada foram avaliadas logo após a EEJ, enquanto que as análises microscópicas (motilidade, vigor e integridade espermática) foram conduzidas em todas as fases do experimento, em microscópio óptico (NIKON, Modelo Eclipse E400, Japão). Motilidade (\%) e vigor espermáticos (0-5) foram avaliados subjetivamente. Para análise da integridade espermática, foram adicionados $5 \mu \mathrm{L}$ de eosina $1 \% \mathrm{e}$ $5 \mu \mathrm{L}$ de nigrosina $1 \%$ a $5 \mu \mathrm{L}$ de sêmen diluído em lâmina pré-aquecida sob mesma temperatura de incubação. Após cinco segundos, era realizado o esfregaço, que secava sob temperatura ambiente (VALLE, 2007). Foram contados 100 espermatozoides e classificados como vivos (não corados) ou mortos (corados).

A integridade espermática das frações líquida e coagulada foi avaliada a cada uma hora durante as seis primeiras horas e às 24 horas de incubação em banho-maria. O coágulo seminal foi fragmentado com auxílio de uma pipeta durante a incubação objetivando acelerar a liquefação, que foi avaliada nas primeiras seis horas, na $12^{\mathrm{a}}$ hora e após as 24 horas de incubação. Após a $24^{a}$ hora, foi mensurada a concentração espermática das duas frações seminais separadamente em câmara de Neubauer, a uma diluição de 1:1 em formol salino $10 \%$.

Análise estatística

A análise estatística foi realizada no programa StatView versão 5.0 (SAS Institute Inc., Cary, NY, USA), com todos os dados expressos como média \pm EPM (erro padrão da média). Concentração e volume médio da fração líquida e coagulada foram comparados pelo teste t pareado. A avaliação do efeito da temperatura e dos tempos de incubação sobre a integridade espermática foi realizada por ANOVA. Todos os testes foram aplicados a 5\% de probabilidade.

\section{RESULTADOS E DISCUSSÃO}

A tricotomia do saco escrotal não comprometeu a quantidade e qualidade do ejaculado, provavelmente devido ao fato de a pelagem nessa região ser bastante escassa e, aparentemente, não exercer influência relevante na manutenção da temperatura escrotal. Além disso, não houve casos de lesões cutâneas ou dermatites em função da tricotomia.

A fração líquida obtida se apresentava viscosa e translúcida, e a fração coagulada apresentava aspecto gelatinoso, coloração esbranquiçada e opaca, era consistente e de difícil manipulação. Os volumes médios da fração líquida e coagulada foram, respectivamente, de $0,20 \pm 0,10 \mathrm{~mL}$ e $0,20 \pm 0,02 \mathrm{~mL}$. A concentração espermática do coágulo $\left(1,1 \pm 0,3 \times 10^{8}\right.$ espermatozoides $\mathrm{mL}^{-1}$ ), após a sua liquefação, foi superior a da fração líquida $\left(1,3 \pm 0,9 \times 10^{7}\right.$ espermatozoides $\left.\mathrm{mL}^{-1}\right)(\mathrm{P}=0,04)$. Esses dados corroboram afirmativas anteriores de que a concentração espermática é consideravelmente maior na fração coagulada que na fração líquida do ejaculado de C. apella (NAGLE \& DENARI, 1983; PAZ et al., 2006a; ARAÚJO et al., 2009).

Em uma amostra de fração líquida, foram verificados espermatozoides com motilidade $20 \%$ e vigor 4, que cessou 40 minutos após a ejaculação. BARNABE et al. (2002) analisaram a fração líquida do sêmen fresco de C. apella, coletado por EEJ, e obtiveram motilidade média de $68,4 \%$ e vigor 2,6 . PAZ et al. (2006b) observaram $24 \%$ de motilidade e vigor de 1,6 na análise da fração coagulada em meio TCM 199 acrescido de tripsina e hialuronidase. O vigor verificado no presente trabalho foi superior, e a motilidade foi equivalente à relatada por ARAÚJO et al. (2009) na diluição do sêmen de $\boldsymbol{C}$. apella em $\mathrm{ACIN}$, a $37^{\circ} \mathrm{C}$. Não foram observados espermatozoides móveis na fração coagulada. Contudo, a ausência de motilidade espermática na fração coagulada não inviabiliza a sua utilização, uma vez que determinadas técnicas de fecundação oocitária, como a injeção intracitoplasmática de células espermáticas (ICSI), não exigem a utilização de espermatozoides móveis (NG et al., 2002).

O coágulo seminal de $\boldsymbol{C}$. apella não se liquefaz espontaneamente (NAGLE \& DENARI, 1983). NAGLE \& DENARI (1983) e PAZ et al. (2006b) recomendam a liquefação enzimática do coágulo em tripsina ou hialuronidase. Entretanto, o uso de enzimas proteolíticas, além de não promover a liquefação total, pode causar danos às membranas espermáticas, impedindo o sucesso da criopreservação (HERNÁNDEZ-LÓPEZ et al., 2002; PAZ et al., 2006b). Neste estudo, o coágulo seminal se liquefez sem qualquer tratamento enzimático, apenas por meio de suaves e contínuos "cortes" realizados com pipeta durante a incubação. Foi observado que a liquefação do coágulo iniciou, em média, a partir da 0 hora; na $12^{\text {a }}$ hora de incubação, a maior parte do coágulo estava liquefeita; e na $24^{a}$ hora havia ocorrido a liquefação total, independentemente da temperatura aplicada, quando era possível avaliar a concentração espermática da fração coagulada a partir de sua diluição total. A ACP- $118^{\circledR}$ não apresentou a mesma eficiência que a ACIN na liquefação do coágulo. ARAÚJO et al. (2009), ao utilizarem a $\mathrm{ACIN}$, atingiram a liquefação total a $37^{\circ} \mathrm{C}$, 
em média, entre a $1^{\text {a }}$ e a $2^{\text {a }}$ hora de incubação, sem adição de enzimas, porém realizando a fragmentação mecânica do coágulo de forma mais frequente que neste estudo, sendo possível concluir que o coágulo se reagrupa rapidamente quando em repouso e absorve grande número de espermatozoides do ejaculado conforme proposto por HERNÁNDEZ-LÓPEZ et al. (2008).

Com relação à manutenção da integridade espermática do sêmen diluído em ACP-118 ${ }^{\circledR} \mathrm{e}$ conservado em banho-maria, foi observado que, imediatamente após a diluição (0 hora) e nas duas horas iniciais, a temperatura de $37^{\circ} \mathrm{C}$ demonstrou ser o melhor tratamento, sendo verificados $51,5 \pm 5 \%$ de espermatozoides vivos na 0 hora; $45,8 \pm 8 \%$ na $1{ }^{\text {a }}$ hora; e $42,7 \pm 5 \%$ na $2^{\mathrm{a}}$ hora de incubação (Figura 1 ).

Nas horas seguintes (da $3^{\mathrm{a}}$ a $6^{\mathrm{a}}$ hora), não houve diferença estatística entre as temperaturas. Após 24 horas de incubação, o melhor tratamento observado foi a $33^{\circ} \mathrm{C}$, com $47 \pm 12,8 \%$ de espermatozoides íntegros, seguido das temperaturas $37^{\circ} \mathrm{C}(15,5 \pm 3,6 \%)$ e $35^{\circ} \mathrm{C}$

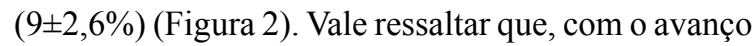
do tempo de incubação, houve um aumento expressivo do percentual de espermatozoides íntegros em razão da maior liquefação do coágulo seminal, o que resultou na liberação de mais espermatozoides vivos do coágulo para o diluente.

ARAÚJO et al. (2009) relatam 72,0 $\pm 3,0 \%$ de integridade espermática no sêmen de $\boldsymbol{C}$. apella diluído em ACIN após sete horas de incubação a $37^{\circ} \mathrm{C}$.
Portanto, não é possível comparar o desempenho da ACIN com o da ACP-118 ${ }^{\circledR}$ após 24 horas de incubação nas três temperaturas avaliadas. No entanto, vale ressaltar que a ACP- $118^{\circledR}$ manteve uma grande quantidade de espermatozoides vivos após 24 horas de incubação. Dessa forma, o diluente à base de ACP$118^{\circledR}$ a $33^{\circ} \mathrm{C}$, além de ser de fácil preparo e manutenção em condições de campo e promover a liquefação do coágulo seminal de $\boldsymbol{C}$. apella, pode ser utilizado para conservação dos espermatozoides quando há necessidade de transporte do ejaculado por um período de até 24 horas. Isso possibilita a coleta do sêmen no habitat do animal e o transporte até o laboratório, facilitando a formação de bancos de germoplasma de animais de vida livre.

\section{CONCLUSÃO}

$\mathrm{O}$ diluente à base de $\mathrm{ACP}-118^{\circledR}$ é eficiente na liquefação do coágulo seminal e na manutenção da integridade espermática a 33,35 e $37^{\circ} \mathrm{C}$ até 24 horas após a eletroejaculação de $\boldsymbol{C}$. apella.

\section{AGRADECIMENTOS}

Ao Centro Nacional de Primatas (IEC/SVS/MS), ao Instituto Evandro Chagas e ao $\mathrm{CNPq}$, pelos recursos humanos e financeiros, e à ACP Biotecnologia ${ }^{\mathbb{B}}$ (Fortaleza - CE), pelo fornecimento do ACP-118 ${ }^{\circledR}$.

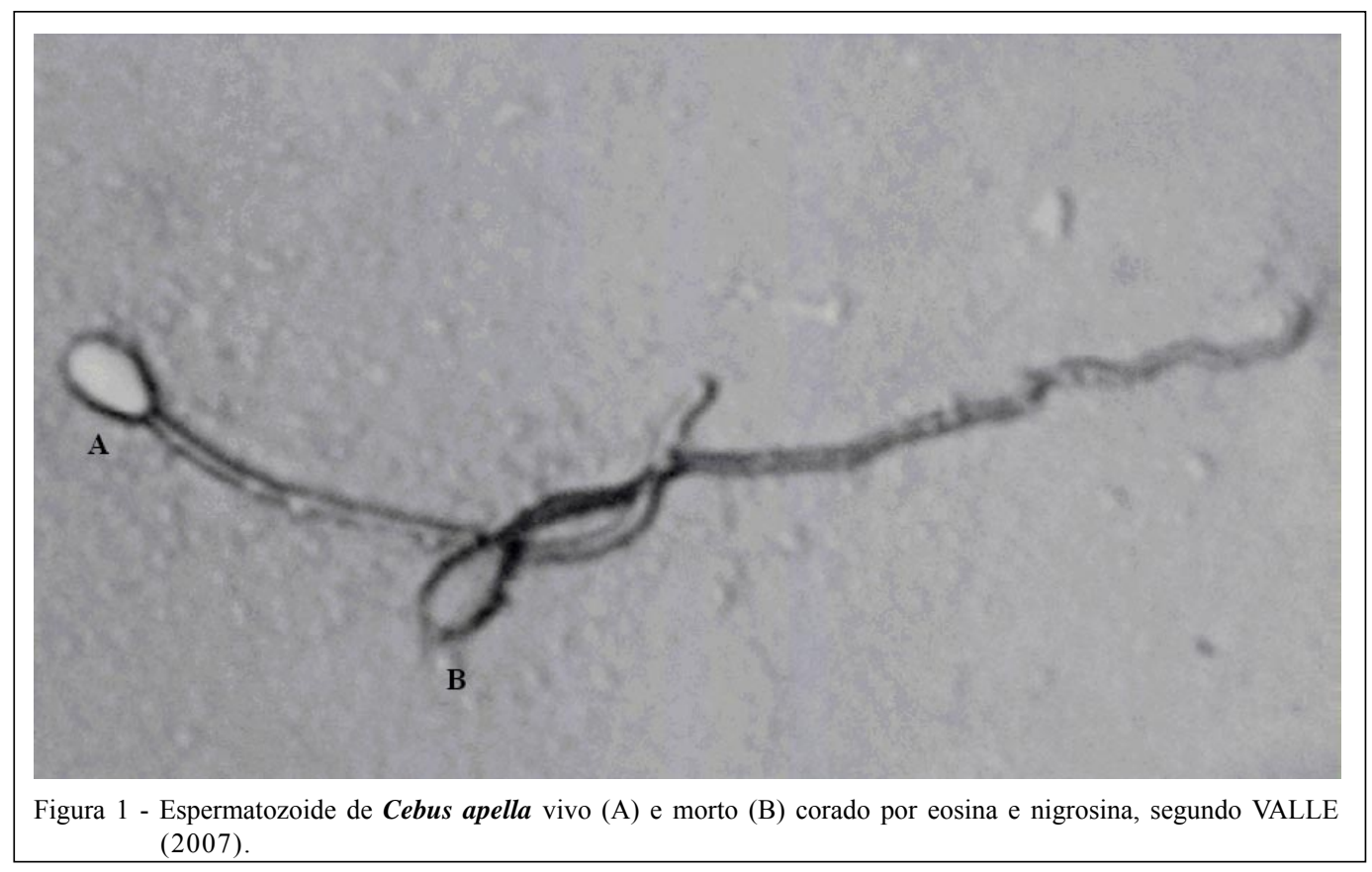

Ciência Rural, v.40, n.3, mar, 2010. 


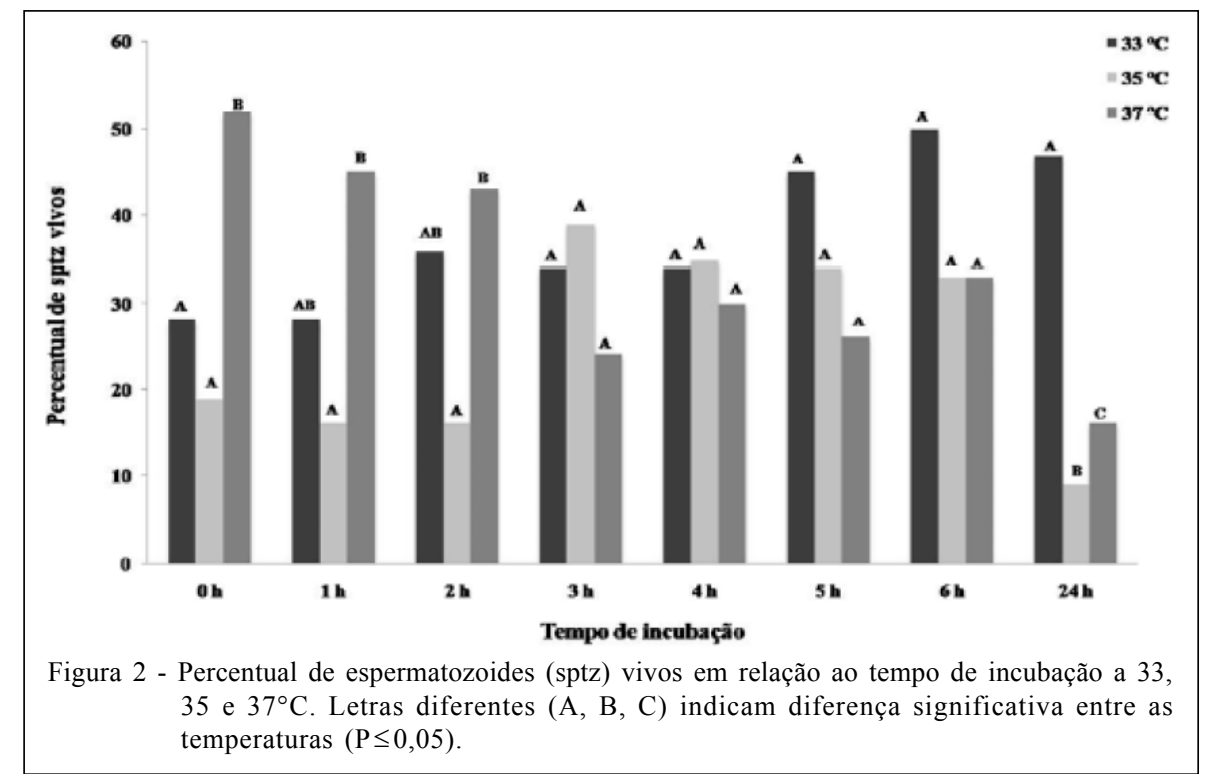

\section{COMITÊ DE ÉTICA E BIOSSEGURANÇA}

Todos os procedimentos realizados foram aprovados pelo Comitê de Ética em Pesquisa com Animais do Instituto Evandro Chagas (CEPAN-IEC-SVS-Protocolo 0011/ 2007).

\section{REFERÊNCIAS}

ARAÚJO, L.L. et al. Avaliação do uso de solução à base de água de coco a $37^{\circ} \mathrm{C}$ para diluição de sêmen de Cebus apella (macacoprego) mantido em cativeiro. Ciência Animal Brasileira (UFG), v.10, n.2, p.588-594, 2009. Disponível em: <http:// www.revistas.ufg.br/index.php/vet/article/view/2739/4851>. Acesso em: 12 set. 2009.

BARNABE, R.C. et al. Analysis of some normal parameters of the spermiogram of captive capuchin monkeys (Cebus apella Linnaeus, 1758). Brazilian Journal of Veterinary Research and Animal Science, v.39, n.6, p.331-333, 2002 Disponível em: <http://www.scielo.br/pdf/bjvras/v39n6/ 15850.pdf $>$. Acesso em: 15 dez. 2009. doi: 10.1590/S141395962002000600010

CARDOSO, R.C.S. Características in vitro do espermatozóide canino criopreservado em água de coco. 2005. 197f. Tese (Doutorado em Reprodução e Sanidade Animal) - Curso de Pós-graduação em Ciências Veterinárias Universidade Estadual do Ceará, Fortaleza, CE.

DOMINGUES, S.F.S.; CALDAS-BUSSIERE, M.C. Fisiologia e biotécnicas da reprodução desenvolvidas em fêmeas de Primatas Neotropicais importantes para a pesquisa biomédica. Revista Brasileira de Reprodução Animal, v.30, n.1, p.57-71, 2006. Disponível em: <http://www.cbra.org.br/pages/publicacoes/rbra/ download/RB054\%20Domingues\%2057-71.pdf >. Acesso em: 16 dez. 2009.

DOMINGUES, S.F.S. et al. Ultrasonographic imaging of the reproductive tract and surgical recovery of oocytes in Cebus apella (capuchin monkey). Theriogenology, v.68, n.9, p.12511259, 2007. Disponível em: <http://www.theriojournal.com/ article/S0093-691X(07)00526-2/abstract>. Acesso em: $15 \mathrm{dez}$. 2009. doi:10.1016/j.theriogenology.2007.08.023.

HERNÁNDEZ-LÓPEZ, L. et al. Seasonal emission of seminal coagulum and in vivo sperm dynamics in the Black-handed
Spider Monkey (Ateles geoffroyi). Theriogenology, v.69, p.466-472, 2008. doi:10.1016/j.theriogenology.2007.10.016.

HERNÁNDEZ-LÓPEZ, L. et al. Sperm quality differences between the rainy and dry seasons in captive black-handed spider monkeys (Ateles geoffroyi). American Journal of Primatology, v.57, n.1, p.35-41, 2002. doi: 10.1002/ajp.1086.

IUCN (International Union for Conservation of Nature), 2008 Acessado em: 16 maio 2009. Online. Disponível em: $<$ http:/ /cms.iucn.org $>$.

LEÃO, D.L. et al. Quantitative analysis of spermatogenesis in capuchin monkey (Cebus apella). Brazilian Journal of Morphological Sciences, v.25, n.1, p.35, 2008. Disponível em: <http://200.152.208.135/sba bjms/media/revistas/v25n14a02.pdf>. Acesso em: 16 dez. 2009.

NAGLE, C.A.; DENARI, J.H. The Cebus Monkey (Cebus apella). In: HEARN, J.P. Reproduction of new world primates. Lancaster: MTP, 1983. p.149-179.

NG, S.C. et al. Intracytoplasmatic injection of frozen-thawed epididymal spermatozoa in a nonhuman primates model, the cynomolgus monkey (Macaca fascicularis). Theriogenology, v.58, p.1385-1397, 2002

PAZ, R.C.R. et al. Avaliação das características seminais de macacos pregos (Cebus apella) mantidos em cativeiro, antes e após vasectomia bilateral. Brazilian Journal of Veterinary Research and Animal Science, v.43, n.4, p.561-567, 2006a. Disponível em: <http://www.fumvet.com.br/novo/revista/43/n4/ 561-567.pdf $>$. Acesso em: 16 dez. 2009.

PAZ, R.C.R. et al. O efeito das enzimas hialuronidase e tripsina na liquefação do sêmen de macacos pregos (Cebus apella). Brazilian Journal of Veterinary Research and Animal Science, v.43, n.2, p.196-201, 2006b. Disponível em: <http:/ /www.revistasusp.sibi.usp.br/pdf/bjvras/v43n2/07.pdf>. Acesso em: 15 dez. 2009.

VALLE, R.R. Colheita, análise e criopreservação de sêmen de uma espécie modelo de primata neotropical, sagui-de-tufo-branco (Callithrix jacchus). 2007. 535f. Tese (Doutorado em Reprodução Animal) - Curso de Pós-graduação em Reprodução Animal, Universidade de São Paulo, São Paulo, SP. 